\title{
Dynamical Optimization and Synchronization in Adaptive Complex Networks
}

\author{
Maoyin Chen and Jürgen Kurths
}

\begin{abstract}
We introduce two dynamical optimization coupling mechanisms for getting different kinds of synchronization in adaptive complex networks. At each node in the network there is an oscillator and the ensemble of oscillators could be either identical or non-identical. For each oscillator, we adjust only one incoming link's strength in different time intervals while the other incoming links' strengths remain constant. The dynamical optimization coupling mechanisms are in effect "winner-take-all" strategies. If one incoming link for each oscillator has the maximal competition ability in different time intervals, its strength increases by a small value. This way, we realize different kinds of synchronization in adaptive complex networks with undelayed or delayed couplings, as well as ensure that all oscillators have uniform intensities during the transition to synchronization. We also enhance the synchronizability in complex networks with identical oscillators.
\end{abstract}

\section{Introduction}

Real-world complex networks (CNs) are interacting dynamical entities with an interplay between dynamical states and interaction patterns. While topological studies have revealed important organization principles in the structures [1-6], a more complete understanding would require characterizations beyond the topology. There are recently several approaches in this direction. Especially, (i) Intensive investigations of synchronization dynamics in oscillatory networks [9,17-19,23-26,32-34,37-40.]. However, most of these works consider networks that do not change the topology

Maoyin Chen

Tsinghua National Laboratory for Information Science and Technology, Department of Automation, Tsinghua University, Beijing 100084, P. R. China. e-mail: mychen@mail.tsinghua.edu.cn

Jürgen Kurths

Center for Dynamics of Complex Systems, Potsdam Universität, Am Neuen Palais 10, D-14469,

Germany. e-mail: jkurths@gmx.de 
with the dynamics. (ii) Growing attention on unified studies of the coevolution of dynamical states and network structures [10-15,21,22,27-31,42,43]. Models of adaptive complex networks (ACNs) have been proposed, e.g., evolving of oscillators due to fitness in interacting species [10], reinforcement of connection strength [11] or rewiring of links [12] due to payoffs among agents playing games; or adaptive changes of coupling strength according to the state distance in globally coupled chaotic maps [13] in a desynchronized regime

ACNs appear in many biological applications. They combine topological evolution of the network with dynamics in the network oscillators. Recently, Gross and Blasius provided a survey on adaptive coevolutionary networks [15]. According to this survey, the majority of recent studies revolve around two key questions corresponding to two distinct lines of research: i) what are the values of important topological properties of a network that is evolving in time and, ii) how does the functioning of the network depend on these properties? The first line of research is concerned with the dynamics of networks [15]. Here the topology of the network itself is regarded as a dynamical system. It changes in time according to specific, often local, rules. Investigations in this area have revealed that certain evolution rules give rise to peculiar network topologies with special properties. The dynamics of ACNs has been investigated in a number of parallel studies from different fields, ranging from genomics to game theory. The second major line of network research focuses on the dynamics on networks [15]. Here each oscillator of the network represents a dynamical system. The individual systems are coupled according to the network topology. Thus, the topology of the network remains static, while the states of the oscillators change dynamically. Important processes that are studied within this framework include synchronization of the individual dynamical systems [9,14,17-19,21-34,37-40,42,43], and contact processes, such as opinion formation and epidemic spreading [44-46].

As a typical dynamical regime on networks, synchronization, especially the ability of networks to obtain synchronization (synchronizability), attracts lots of interests [14,17-19,21-33,37-40,42,43]. Complete synchronization (CS) in networks of identical oscillators [27-30] and phase synchronization (PS) in networks of nonidentical oscillators $[21,22]$ can be ensured by introducing adaptive local couplings between connected oscillators, or adaptive global couplings in the whole networks. The networks due to adaptive couplings are also a kind of ACNs. Based on the local dynamical neighborhood information in networks with identical oscillators, Zhou and Kurths introduced an adaptive coupling scheme [27]. For simplicity, this method is called the Zhou-Kurths method. Consequently, the adaptive self-organization by the Zhou-Kurths method drives the network into the direction of a more homogeneous topology, ongoing with an enhanced ability for synchronization. Hence it is possible to synchronize networks that exceed by several orders of magnitude the size of the largest comparable random graph that is still synchronisable [15].

However, there are some shortcomings in these studies on ACNs, where the local or global couplings are changed adaptively. The first one is that these works can not ensure that all oscillators have uniform intensities during the transition to synchronization. From the works on synchronizability in networks with a given topology, 
the synchronizability becomes optimal when the intensities become uniform in networks. This can be verified by the load [37,38] and degree [39] based weighted networks. For randomly enough unweighted and weighted networks, the synchronizability is controlled by $S_{\max } / S_{\min }$, where $S_{\max }$ and $S_{\min }$ are the maximum and minimum of intensities $S_{i}$, defined by the sum of the couplings for oscillator $i$ [32]. For scale-free (SF) networks [32], one gets $S_{\max } / S_{\min }=k_{\max } / k_{\min } \sim N^{1 / 2}$, where $k_{\max }$ and $k_{\min }$ are the maximal and minimal degrees, respectively. For a fixed network topology, the synchronizability can be enhanced if the intensities become more homogeneous. The second problem is that these methods can not be effectively applied to networks with delayed couplings. For example, for networks with identical chaotic oscillators, the non-uniformity of intensities does not ensure the existence of a synchronous manifold in networks with delayed couplings. Further, there exists no unifying adaptive coupling scheme to get different kinds of synchronization. The scheme for PS in the Kuramoto model can not be effectively applied to PS in networks with non-identical chaotic oscillators and CS in networks with identical chaotic oscillators. The scheme for CS in networks with identical chaotic oscillators can not be effectively applied to PS in networks with non-identical oscillators.

In this chapter we develop two adaptive coupling schemes to get different kinds of synchronization in networks, as well as to ensure that all oscillators have uniform intensities during the transition to synchronization. This chapter is organized as follows. In the next section, we consider PS in the famous Kuramoto model with delayed couplings and external noises. By adaptively adjusting the couplings according to the dynamical gradient network (DGN) approach [22], we ensure PS in different variants of the Kuramoto model, as well as all oscillators have uniform intensities. This approach can be also applied to networks with non-identical oscillators, provided that the definition of 'phase' is well-defined. Furthermore, this approach can be extended to CS in networks with identical oscillators. In section 3 , we further propose another more effective coupling mechanism, the dynamical optimization (DO) mechanism [42,43], for realizing CS in networks with identical oscillators. Though there exist delayed couplings in networks, we realize CS effectively, as well as the intensities for all oscillators are uniform. We also discuss the enhanced synchronizability in scale-free (SF) networks and small-world (SW) networks, due to the DO mechanism. This approach is also applicable to PS in networks with non-identical oscillators. In the last section we draw up our conclusion.

\section{PS in the Kuramoto model}

Among many models that have been proposed to address synchronization phenomena, one of the most successful models is the Kuramoto model [7-9]. It can be used to understand the emergence of synchronization in networks of oscillators. In particular, this model presents a second-order phase transition from incoherence to synchronization. For synchronization in the Kuramoto model, many works assumed that the couplings between connected oscillators are constant [24-26]. Recently, some 
works introduced adaptive couplings in this model. Maistrenko et al. introduced the mechanism of plasticity to study multistability, and assumed that the couplings are varied in accordance with the spike timing-dependent plasticity [20]. Ren and Zhao also proposed adaptive couplings by introducing continuously adaptive couplings, and this can enhance the synchronization in the Kuramoto model. In this scheme, the couplings grow stronger for pairs which have larger phase incoherence [21].

Based on a DGN approach, we also consider synchronization in the Kuramoto model with adaptive couplings. This study is motivated in part by the work $[35,36]$, where the concept of gradient networks is introduced. Gradient networks are directed subnetworks of an undirected "substrate" network in which each oscillator has an associated scalar potential and one outlink that points to the oscillator with the smallest (or largest) potential in the reunion of itself and its neighbors on the substrate network. The existence of gradients has been shown to play an important role in biological transport processes, such as cell migration: chemotaxis, haptotaxis, and galvanotaxis. Naturally, the same mechanism will generate flows in complex networks as well [36]. In addition, gradient networks have been already utilized to enhance synchronization in networks [23]. A general weighted asymmetrical network is regarded as a superposition of a weighted symmetrical network and a weighted gradient network. Depending on the degrees of oscillators, a weighted coupling scheme is proposed to enhance the synchronizability in networks. However, the proposed gradient network is static, i. e., its structure is time independent. Differing from the static gradient networks in Ref. [23], gradient networks developed in this section are dynamical, which implies that the gradient networks in different time intervals are different.

Here the Kuramoto model consists of a population of $N$ coupled oscillators where the phase $\theta_{i}(t)$ of the $i$-th oscillator evolves in time according to

$$
\frac{d \theta_{i}}{d t}=w_{i}+\sum_{j} W_{i j} A_{i j} \sin \left(\theta_{j}-\theta_{i}\right), i=1,2, \cdots, N
$$

where $w_{i}$ are natural frequencies distributed with a given probability density $g(w)$, $A_{i j}$ is the binary adjacency matrix representing the topology of networks, and it is not necessary symmetric. Further, $W_{i j} \geq 0$ is the coupling strength of the incoming link $(i, j)$ pointing from oscillator $j$ to oscillator $i$ if they are connected. Denote $K_{i}$ as the index set of neighbors of oscillator $i$.

The Kuramoto model (1) can be solved in terms of the order parameter $r(t)$ that measures the extent of synchronization as

$$
r(t) \mathrm{e}^{\zeta \Psi(t)}=\frac{1}{N} \sum_{j=1}^{N} \mathrm{e}^{\zeta \theta_{j}(t)}
$$

where $\zeta^{2}=-1, \Psi(t)$ stands for an average phase, and the parameter $0 \leq r(t) \leq 1$. Obviously, if $r(t)=1$, PS in the Kuramoto model (1) is realized. The parameter $r(t)$ given by Eq. (2) has been widely used [7-9,21,25]. 
We first introduce adaptive couplings into the Kuramoto model (1). In order to do so, we segment the time interval $\left[t_{0},+\infty\right)$ into

$$
\left[t_{0},+\infty\right)=\bigcup_{n \geq 1}\left[t_{n-1}, t_{n}\right),
$$

where $t_{n}=t_{0}+n T, t_{0}$ is the transient time, the length $T$ of intervals is chosen suitably. For the parameter $r(t)$, we define one local order parameter for oscillator $i$ in the interval $\left[t_{n-1}, t_{n}\right)$ :

$$
r^{i, n}=\frac{1}{T} \int_{t_{n-1}}^{t_{n}} r_{i}(t) d t
$$

with

$$
r_{i}(t) \mathrm{e}^{\zeta \Psi_{i}(t)}=\frac{1}{k_{i}+1} \sum_{j \in K_{i} \cup\{i\}} \mathrm{e}^{\zeta \theta_{j}(t)},
$$

where $k_{i}$ is the degree of oscillator $i$. The parameter $r^{i, n}$ can measure the local synchronization extent among oscillator $i$ and its neighbors. If $r^{i, n_{0}}=1$ for certain $n_{0}$, oscillator $i$ and its neighbors are locally synchronized in the interval $\left[t_{n_{0}-1}, t_{n_{0}}\right)$.

For the network of oscillators, the extent of synchronization is to choose the order parameter $r_{0}(n)$ :

$$
r_{0}(n):=\frac{1}{T} \int_{t_{n-1}}^{t_{n}} r(t) d t
$$

If there is a $n_{0}$ such that $r\left(n_{0}\right)=1$, we conclude that synchronization in the network is realized effectively.

Now we introduce an adaptive coupling scheme into the Kuramoto model. Our idea to adjust the coupling $W_{i j}$ in the interval $\left[t_{n}, t_{n+1}\right)$ is based on the concept of gradient networks $[35,36]$. To define a gradient network at the instant $t=t_{n}$, we consider a network denoted by $\Sigma=\left(V, E_{n}\right)$, where $V$ stands for the set of oscillators, and $E_{n}$ denotes the set of links at the instant $t=t_{n}$. Consider a field denoted by $h^{n}=\left\{h_{1}^{n}, \cdots, h_{N}^{n}\right\}$ at the instant $t=t_{n}$, where $h_{i}^{n}$ is the scalar assigned to oscillator $i$. We define the gradient $\nabla_{h_{i}^{n}}$ of the field $h_{i}^{n}$ in oscillator $i$ to be the directed link $\nabla_{h_{i}^{n}}=\left(i, \mu_{i}^{n}\right)$, where $\mu_{i}^{n} \in K_{i}$ represents one neighbor of oscillator $i$. At the instant $t=t_{n}$, the network $\Sigma_{g}=\left(V, \nabla_{n}\right)$, where $\nabla_{n}$ is the set of the gradients $\nabla_{h_{i}^{n}}$, is called a gradient network. Note that at different time instants the gradient networks can be different. In this section, this kind of gradient networks is called DGNs. In the gradient network $\Sigma_{g}$, the directed link $\left(i, \mu_{i}^{n}\right)$ points from oscillator $\mu_{i}^{n}$, at which the scalar field has the minimum (or maximum) value in oscillator $\mu_{i}^{n} \in K_{i}$, i.e. [36]

$$
\mu_{i}^{n}=\arg \max _{j \in K_{i}}\left\{-h_{j}^{n}\right\}
$$

to oscillator $i$. If several neighbors have the same scalar field, we choose only one randomly. For oscillator $i$ in the Kuramoto model (1), we choose the scalar field $h_{i}^{n}$ as

$$
h_{i}^{n}=r^{i, n}
$$


Denote the coupling $W_{i j}$ in the interval $\left[t_{n-1}, t_{n}\right)$ as $W_{i j}^{n}$. In the gradient network composed of the gradients $\nabla_{h_{i}^{n}}$, we adjust the coupling $W_{i \mu_{i}^{n}}$ of the incoming link $\left(i, \mu_{i}^{n}\right)$ pointing from oscillator $\mu_{i}^{n}$ to oscillator $i$. In the interval $\left[t_{n}, t_{n+1}\right)$, we adaptively adjust the coupling $W_{i \mu_{i}^{n}}$ of the incoming link $\left(i, \mu_{i}^{n}\right)$ in the gradient network $\Sigma_{g}=\left(V, \nabla_{n}\right)$ by

$$
W_{i \mu_{i}^{n}}^{n+1}:=W_{i \mu_{i}^{n}}^{n}+\varepsilon,
$$

where $\varepsilon>0$ is an arbitrary small incremental coupling. When the link $(i, j)$ does not belong to the gradient network $\Sigma_{g}$, its coupling satisfies

$$
W_{i j}^{n+1}:=W_{i j}^{n} .
$$

From Eqs. (6-9), the DGN approach is also a dynamical optimization coupling scheme. It reflects the "winner-take-all" strategy in the sense of scale fields. For oscillator $i$, the incoming link to be adjusted is always chosen as one pointing from one neighborhood oscillator with the minimal (or maximal) field to itself. Further, we only adjust one incoming link's strength in different time intervals while the other incoming links' strengths remain constant. Here we define the intensity $S_{i}$ for oscillator $i$ as $S_{i}=\sum_{j \in K_{i}} W_{i j} A_{i j}$. Note that the intensities of all oscillators in networks are uniform, since at each step the intensity of each oscillator increases by the same amount $\varepsilon$.

Now we analyze the feasibility of the above coupling scheme by the linearized dynamics of the Kuramoto model (1). When the Kuramoto dynamics is close to the attractor, the phase differences are small, and then the sine coupling function can be approximated linearly. Therefore, in the interval $\left[t_{n}, t_{n+1}\right)$, the linearized dynamics of oscillator $i$ can be written in the form

$$
\frac{d \theta_{i}}{d t}=\sum_{j} W_{i j}^{n} A_{i j}\left(\theta_{j}-\theta_{i}\right)+\varepsilon\left(\theta_{\mu_{i}^{n}}-\theta_{i}\right) .
$$

In the above equation the last term $\varepsilon\left(\theta_{\mu_{i}^{n}}-\theta_{i}\right)$ is equivalent to the term $-\varepsilon\left(\theta_{i}-\right.$ $\left.\theta_{\mu_{i}^{n}}\right)$, which can be regarded as a negative feedback term for the unidirectional synchronization from oscillator $\mu_{i}^{n}$ to oscillator $i$. This could make the phase difference between oscillator $i$ and its neighbor $\mu_{i}^{n}$ be smaller, which may result in synchronization in the Kuramoto model.

The adaptive scheme (6-9) can be easily extended to Kuramoto models with delayed couplings and external noise. One case is the Kuramoto model described by $[8,24]$

$$
\frac{d \theta_{i}}{d t}=w_{i}+\sum_{j} W_{i j} A_{i j} \sin \left(\theta_{j}-\theta_{i}\right)+\xi_{i}(t), i=1,2, \cdots, N,
$$

where $\xi_{i}(t)$ is white noise due to some complicated environment with expectation and variance

$$
<\xi_{i}(t)>=0,<\xi_{i}(t) \xi_{j}\left(t^{\prime}\right)>=2 \delta_{i j} \delta\left(t-t^{\prime}\right) .
$$

Another case is the Kuramoto model given by [8] 

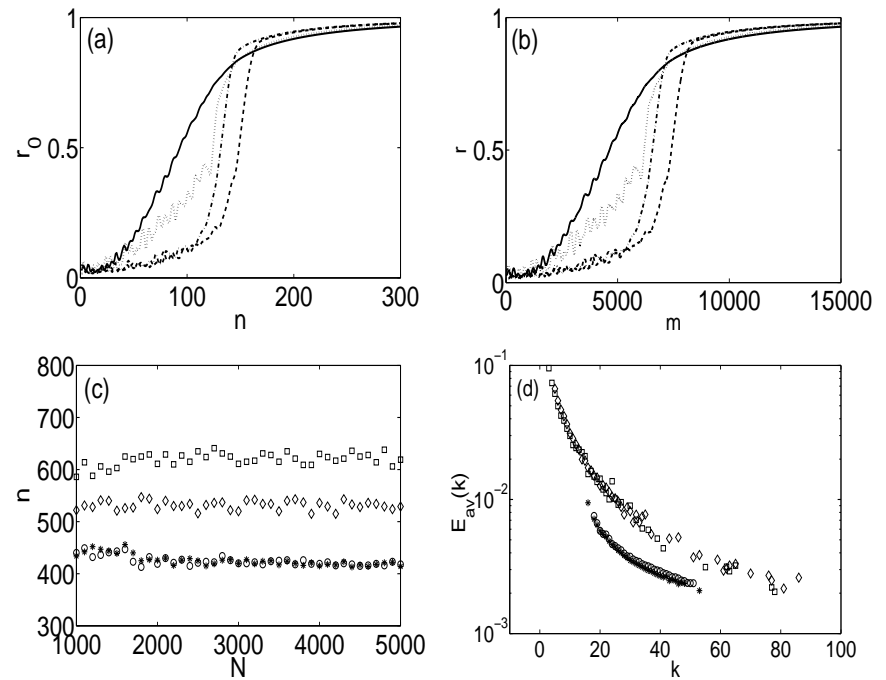

Fig. 1 Simulation results in the Kuramoto model (1) without noise. The parameter $r_{0}(n)$ as a function of the adjustment step $n$ (a), and the parameter $r(t)$ as a function of the step $m$ for solving the Kuramoto models (b), in SF networks (solid line: $M=3$; dotted line: $M=5$ ) and SW networks (dashdot line: $K=2, p=0.03$; dashed line: $K=4, p=0.03$ ). The adjustment step $n$ as a function of the size $N$ in networks (c), and standard deviation $E_{\mathrm{av}}(k)$ as a function of degree $k$ in SF and SW networks with $N=1000$ (square: $M=3$; diamond: $M=5$; star: $K=2, p=0.03$; circle: $K=4$, $p=0.03)$. All estimates are the results of averaging over 50 realizations.

$$
\frac{d \theta_{i}}{d t}=w_{i}+\sum_{j} W_{i j} A_{i j} \sin \left(\theta_{j, \tau}-\theta_{i}\right)+\xi_{i}(t), i=1,2, \cdots, N
$$

where the term $\theta_{j, \tau}$ represents the delayed phase $\theta_{j}(t-\tau)$, and $\tau$ is a constant time delay.

Our simulations are based on SF and SW networks. SF networks are generated by the Barabási-Albert model [2], where the initial network is a fully connected network with $M$ oscillators, labeled by $i=1, \cdots, M$. At every time step a new oscillator is introduced to be connected to $M$ existing oscillators. The probability that a new oscillator is connected to oscillator $i$ depends on the degree $k_{i}$ of oscillator $i$, namely $\Pi_{i}=k_{i} / \sum_{j} k_{j}$. After repeating for $N-M$ times, a SF network has a degree distribution $P(k) \sim k^{-3}$ and the minimal degree $k_{\min }=M$. SW networks are generated by the Newman-Watts model [16]. The initial network is a $K$-nearest-neighbor coupled network consisting of $N$ oscillators arranged in a ring, with each oscillator $i$ being adjacent to its neighbor oscillators $i \pm 1, \cdots, i \pm K / 2$, and with $K$ being even. Then one adds with probability $p$ a connection between a pair of oscillators.

In our simulations in this section, the initial couplings for all incoming links for each oscillator are zero, the natural frequencies of the oscillators are uniformly distributed in the interval $[-1,1]$, the transient time is $t_{0}=100 \mathrm{~s}$, the length of intervals is $T=1 \mathrm{~s}$, and the incremental coupling is $\varepsilon=0.01$. The solution of networks is re- 

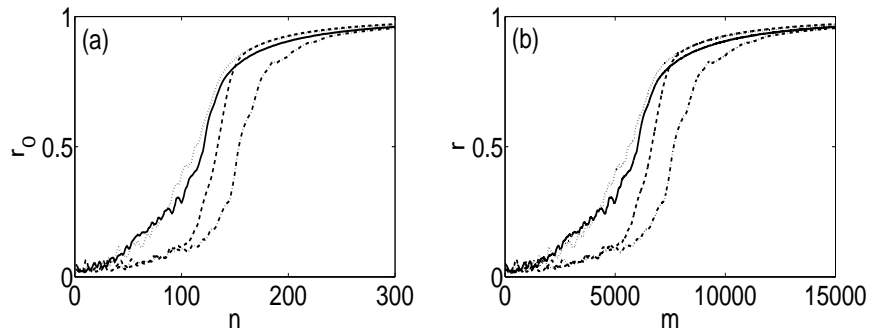

Fig. 2 Simulation results in the Kuramoto model (1) with noise. The parameter $r_{0}(n)$ as a function of $n$ (a), and the parameter $r(t)$ as a function of $m(\mathrm{~b})$, in SF networks (solid line: $M=4$; dotted line: $M=6$ ) and SW networks (dashdot line: $K=6, p=0.01$; dashed line: $K=8, p=0.01$ ). All estimates are the results of averaging over 50 realizations.

solved using the Euler method and the step time $h=0.02 \mathrm{~s}$, and the ending condition for our scheme is $\left|r\left(n_{0}\right)-1\right|<10^{-2}$ for certain $n_{0}$.

We first simulate SF networks with $N=1000$ and SW networks with $N=1000$ and $p=0.03$ in the absence of noise. We plot the local order parameter $r_{0}$ as a function of the adjustment time $n$ [Fig.1 (a)], and the global order parameter $r$ as a function of the step $m(=n / h)$ for solving the Kuramoto model [Fig.1 (b)]. Obviously, due to our coupling scheme $(8,9)$, the Kuramoto model $(1)$ reaches a synchronized regime after several hundreds of adjustment steps. In every time interval, only one incoming link's coupling for each oscillator is adjusted by the same small incremental coupling, and the other incoming links' couplings remain constant. Hence the intensities $S_{i}$ for all oscillators are identical during the transition to synchronization. From Fig.1(a,b), the extent of synchronization in the Kuramoto model increases with increasing of the intensity $S$ given by $S=S_{i}=n \varepsilon$. In our coupling scheme, the intensity $S$ is a good indicator for synchronization in the Kuramoto model. At about $n=300$, namely $S=3$, the Kuramoto model (1) is practically in a synchronized state. However, equal intensities can not be ensured by other known adaptive coupling schemes [21,27]. The intensities in Ref. [27] strongly depend on heterogeneous degrees in SF networks. The larger the degree of an oscillator is, the larger its intensity is.

We also discuss the synchronization in SF and SW networks with different size. Under the same ending condition, we observe that the adjustment steps needed to synchronize SF networks with the same $M$ are almost identical [Fig.1 (c)]. It further means that the time $\left(n_{0} T\right)$ needed to synchronize SF networks with the same $M$ is almost equal. We can also obtain similar results in SW networks with the same $K$ and $p$. The steps in SW networks with the same $p$ and a small $K$ are almost identical while the steps in SF networks with different $M$ are also different. This can be in part explained by the average degree $\langle k\rangle \approx 2 M$ in SF networks and $\langle k>\approx K+(N-1) p / 2$ in SW networks. When the average degree of networks is smaller, it requires a longer time to synchronize networks.

After the ending of our scheme $(8,9)$, we also analyze the relationship between the normalized coupling matrix $G=\left(G_{i j}\right)$ with $G_{i j}=W_{i j}^{n_{0}} A_{i j} / n_{0} \varepsilon$ and the 

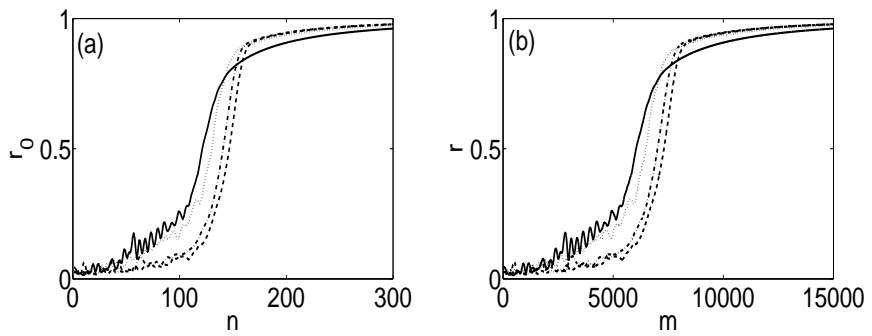

Fig. 3 Simulation results in the Kuramoto model (12) without noise. The parameter $r_{0}(n)$ as a function of $n(\mathrm{a})$, and the parameter $r(t)$ as a function of $m(\mathrm{~b})$, in SF network (solid line: $M=4$; dotted line: $M=7$ ) and SW network (dashdot line: $K=6, p=0.02$; dashed line: $K=8, p=0.02$ ). All estimates are the results of averaging over 50 realizations.

coupling matrix $G_{0}=\left(W_{i j}^{\prime} A_{i j}\right)$ with $W_{i j}^{\prime}=1 / k_{i}$. We compute the average error $E_{\mathrm{av}}(k)=\frac{1}{\gamma_{k}} \sum_{q=1}^{\gamma_{k}} E_{q}$ between $G$ and $G_{0}$, where $\gamma_{k}$ is the number of oscillators with the same degree $k$, and $E_{q}=\sqrt{\sum_{j \neq i}\left(G_{i j}-1 / k_{i}\right)^{2}} / k_{i}$ if $k_{i}=k$. We show that $G_{i j}$ is almost identical to the value $1 / k_{i}$ (or $G_{i j} \sim k_{i}^{-1}$ ) [Fig.1(d)]. After the ending of our scheme, the couplings $W_{i j}^{n_{0}}$ for the incoming links of oscillator $i$ are approximately $n_{0} \varepsilon / k_{i}$. Therefore, for SF networks with the same $M$ and SW networks with the same $K$ and $p$, the maximal coupling relies on the minimal degree in networks. The larger the degree of oscillator $i$ is, the smaller the coupling $W_{i j}^{n_{0}}$ is.

Even if there exists noise in the Kuramoto model (1), we can also obtain similar results in SF networks with different $M$ and SW networks with different $K$ and $p$ [Fig. 2]. For the Kuramoto model (12) with delayed couplings, simulation results are plotted in Fig. $3(\tau=1)$ and Fig. $4(\tau=3)$. Here we only plot figures on the parameters $r_{0}$ and $r$. From these figures, synchronization can be realized effectively.

Note that there are two parameters $T$ and $\varepsilon$ in our scheme. Due to the weak coupling for synchronization in the Kuramoto model, $\varepsilon$ can not be large, but the length $T$ of the intervals can be arbitrarily large. In our simulations $\varepsilon$ can be chosen in the interval [0.0001,0.02]. For different values of $T$ and $\varepsilon$, we obtain similar results.

Remarks: Gómez-Gardeñes et al. proposed another order parameter $r_{\text {link }}$ to measure the extent of synchronization [26], where

$$
r_{\text {link }}=\frac{1}{2 N_{\text {link }}} \sum_{i} \sum_{j \in K_{i}}\left|\lim _{\Delta_{t} \rightarrow \infty} \frac{1}{\Delta_{t}} \int_{t_{r}}^{t_{r}+\Delta_{t}} e^{\zeta\left[\theta_{i}(t)-\theta_{j}(t)\right]} d t\right|,
$$

where $N_{\text {link }}$ is the number of links, $t_{r}$ is a large time. The averaging time $\Delta_{t}$ is taken large enough to obtain good measures of the degree of coherence between each pair of physically connected oscillators. Eqs. $(4,5,7)$ in our scheme can be replaced by

$$
r_{\text {link }}^{i, n}=\frac{1}{k_{i}} \sum_{j \in K_{i}}\left|\frac{1}{T} \int_{t_{n-1}}^{t_{n}} e^{\zeta\left[\theta_{i}(t)-\theta_{j}(t)\right]} d t\right|,
$$



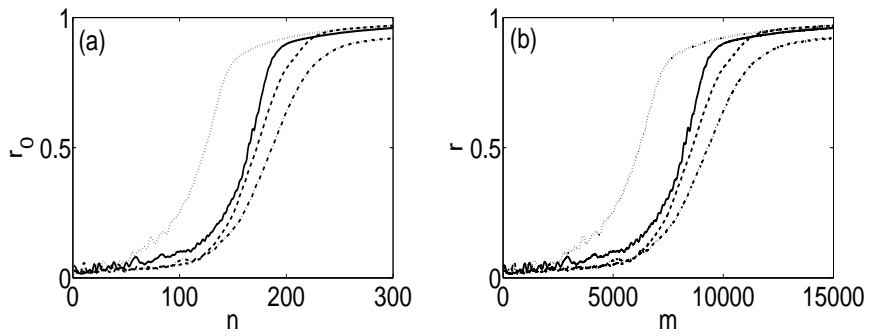

Fig. 4 Simulation results in the Kuramoto model (12) with noise. The parameter $r_{0}(n)$ as a function of $n$ (a), and the parameter $r(t)$ as a function of $m(\mathrm{~b})$, in SF network (solid line: $M=4$; dotted line: $M=6$ ) and SW network (dashdot line: $K=6, p=0.01$; dashed line: $K=8, p=0.01$ ). All estimates are the results of averaging over 50 realizations.

$$
r_{\text {link }}^{\prime}(n)=\frac{1}{2 N_{\text {link }}} \sum_{i} \sum_{j \in K_{i}}\left|\frac{1}{T} \int_{t_{n-1}}^{t_{n}} e^{\zeta\left[\theta_{i}(t)-\theta_{j}(t)\right]} d t\right|
$$

and

$$
h_{i}^{n}=r_{\text {link }}^{i, n},
$$

respectively. One ending condition is $\left|r_{\text {link }}^{\prime}\left(n_{0}\right)-1\right|<10^{-2}$ for certain $n_{0}$. Since numerical results are very similar to those with respect to the parameters $r^{i, n}$ and $r_{0}(n)$ [Figs. 1-4], we omit corresponding figures.

The DGN approach can also be applied to CS in networks with identical oscillators, whose state is represented by $\mathbf{x}_{i}$. In this case, we should assign a suitable scale field to oscillator $i$. Eqs. $(4,5,7)$ in our scheme can be replaced by

$$
\begin{gathered}
r_{\text {link }}^{i, n}=-\frac{1}{k_{i}} \sum_{j \in K_{i}} \frac{1}{T} \int_{t_{n-1}}^{t_{n}}\left\|\mathbf{x}_{i}-\mathbf{x}_{j}\right\| d t, \\
r_{\text {link }}^{\prime}(n)=\frac{1}{2 N_{\text {link }}} \sum_{i} \sum_{j \in K_{i}} \frac{1}{T} \int_{t_{n-1}}^{t_{n}}\left\|\mathbf{x}_{i}-\mathbf{x}_{j}\right\| d t,
\end{gathered}
$$

and

$$
h_{i}^{n}=r_{\text {link }}^{i, n},
$$

respectively. One ending condition is $r_{\text {link }}^{\prime}\left(n_{0}\right)<\varepsilon$ for certain $n_{0}$, and $\varepsilon$ is arbitrary small.

\section{$3 \mathrm{CS}$ and enhanced synchronizability in ACNs}

In this section, inspired by the DGN approach, we develop another more effective optimization coupling mechanism: the DO coupling mechanism. It does not only realize different kinds of synchronization in networks but also leads to enhanced synchronizability in SF and SW networks. In this section, we first consider CS in 
networks with undelayed or delayed couplings. Then we study how to enhance the synchronizability in SF and SW networks.

\subsection{CS in ACNs}

Our general model for networks consisting of $N$ coupled identical chaotic oscillators with a time-varying coupling matrix is given by

$$
\dot{\mathbf{x}}_{i}=\mathbf{F}\left(\mathbf{x}_{i}\right)+\sum_{j=1}^{N} G_{i j} \mathbf{H}\left(\mathbf{x}_{j}, \mathbf{x}_{i}\right)
$$

where $\mathbf{x}_{i}$ is the state, $\mathbf{F}\left(\mathbf{x}_{i}\right)$ is the dynamics of the individual oscillator $\mathbf{x}_{i}, \mathbf{H}\left(\mathbf{x}_{j}, \mathbf{x}_{i}\right)$ is the inner coupling function, $G=\left(G_{i j}\right)$ is the outer coupling matrix. $G_{i j}=W_{i j} A_{i j}$, where $A=\left(A_{i j}\right)$ is the binary adjacency matrix, $W_{i j}$ is the coupling strength of the incoming link $(i, j)$ pointing from oscillator $j$ to oscillator $i$ if they are connected, $G_{i i}=-\sum_{j \in K_{i}} A_{i j} W_{i j}, K_{i}$ is the neighbor set of oscillator $i$.

In this section we consider CS in network (20) in two cases. (i) One case is the network (20) with undelayed couplings, where the function $\mathbf{H}\left(\mathbf{x}_{j}, \mathbf{x}_{i}\right)=\mathbf{H}_{0}\left(\mathbf{x}_{j}\right)-$ $\mathbf{H}_{0}\left(\mathbf{x}_{i}\right)$, and $\mathbf{H}_{0}$ is the output function for each oscillator. (ii) The other case is the network (20) with delayed couplings, in which the function $\mathbf{H}\left(\mathbf{x}_{j}, \mathbf{x}_{i}\right)=\mathbf{H}_{0}\left(\mathbf{x}_{j}(t-\right.$ $\tau))-\mathbf{H}_{0}\left(\mathbf{x}_{i}(t)\right)$ with a time delay $\tau>0$.

In the above section, we have proposed a DGN approach to realize PS in the Kuramoto model, and this approach can be also applied to CS in networks with identical oscillators. However, the DGN approach is very special in two aspects. One is that it should assign a scale potential to each oscillator within any time interval, which depends on the extent of the local synchronization among itself and its neighbor oscillators. The other is that the incoming link to be adjusted by the DGN approach is often not mostly effective. Inspired by the idea of the DGN approach [22], we have further introduced a DO mechanism to SF networks [42]. It also reflects the "winner-take-all" strategy, where the incoming link to be adjusted is always chosen as a pair of oscillators with the weakest synchronization. This means that the DO mechanism is much more effective than the DGN approach.

We first introduce the idea of the DO mechanism. In the interval $\left[t_{n}, t_{n+1}\right)$, the choice of the incoming link for oscillator $i$ is based on the maximal accumulated synchronization error in its neighborhood, rather than depending on the scalar fields of oscillators [22]. The DO mechanism is introduced as follows:

(i) For the incoming link $(i, j)$ of oscillator $i$, we accumulate the synchronization errors by the integral function

$$
E_{n}(i, j)=\int_{t_{n-1}}^{t_{n}} \phi\left(\mathbf{x}_{i}, \mathbf{x}_{j}\right) d t
$$



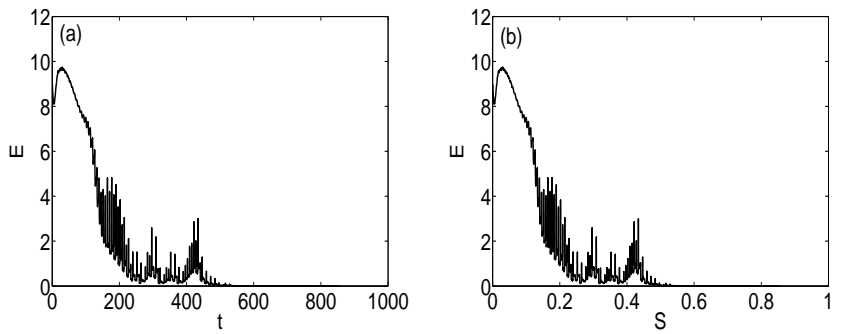

Fig. 5 The average synchronization error $E$ in SF networks with undelayed couplings as a function of (a) time $t$, and (b) intensity $S$, by the DO mechanism. The parameters are $N=1000, M=5$, $T=1 \mathrm{~s}, \varepsilon=0.001$ and $\sigma=1.5$.

where $\phi$ is the error function relying on different kinds of synchronization in networks.

(ii) By the optimization in the neighborhood of oscillator $i$, we identify the incoming link $\left(i, j_{\max }^{n}\right)$ with the index

$$
j_{\max }^{n}=\arg \max _{j \in K_{i}} E_{n}(i, j) .
$$

(iii) We adjust the coupling strength $W_{i j}$ adaptively by

$$
\left\{\begin{array}{l}
W_{i j_{\max }^{n}}^{n+1}:=W_{i j_{\max }^{n}}^{n}+\varepsilon \\
W_{i j}^{n+1}=W_{i j}^{n}, j \neq j_{\max }^{n}
\end{array}\right.
$$

Compared with the incoming link generated by the optimization scheme $(6,7)$, namely the DGN approach, the incoming link generated by the DO mechanism is much more effective. Further, there is one common point: the intensities of the oscillators in the networks are also uniform, since at each step the intensity of each oscillator increases by the same amount $\varepsilon$ during the transition to synchronization.

Our simulations in this section are also based on SF networks generated by the Barabási-Albert model [2] and SW networks generated by the Newman-Watts model [16]. In the following, network (1) is a network of Rössler oscillators: $\mathbf{x}_{i}=\left(x_{i}, y_{i}, z_{i}\right), \mathbf{F}\left(\mathbf{x}_{i}\right)=\left(-0.97 y_{i}-z_{i}, 0.97 x_{i}+0.15 y_{i}, z_{i}\left(x_{i}-8.5\right)+0.4\right)$, the function $\mathbf{H}_{0}\left(\mathbf{x}_{i}\right)=\left(x_{i}, 0,0\right)$, and the error function

$$
\phi\left(\mathbf{x}_{i}, \mathbf{x}_{j}\right)=\left|x_{i}-x_{j}\right|+\left|y_{i}-y_{j}\right|+\left|z_{i}-z_{j}\right| .
$$

In order to verify CS, we define the average synchronization error as

$$
E=\frac{1}{N} \sum_{i=1}^{N}\left\|\mathbf{x}_{i}-\overline{\mathbf{x}}\right\|
$$

where $\overline{\mathbf{x}}=(\bar{x}, \bar{y}, \bar{z})$ is the mean-field of all $\mathbf{x}_{i}$. In our simulations, the initial coupling strengths for all incoming links are zero, the transient time is $t_{0}=100 \mathrm{~s}$, the length of 

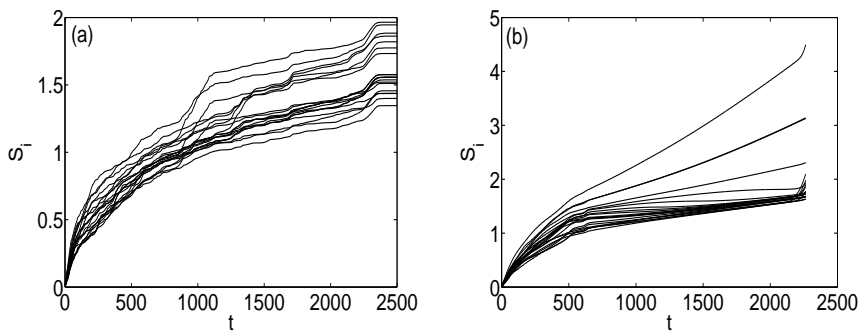

Fig. 6 The intensities $S_{i}$ as a function of time $t$ for arbitrarily 20 oscillators in SW networks with undelayed couplings (a), or delayed couplings (b), by the Zhou-Kurths method. The parameters are $N=500, K=4, p=0.003, \gamma=0.002, \tau=0.01$ s.

time intervals is $T=1 \mathrm{~s}$, and $\varepsilon=0.001$. Further, initial conditions for all oscillators are randomly chosen from the chaotic attractor. The solution of network (20) is solved by using the Euler method with the time step $h=0.01 \mathrm{~s}$, and our ending condition for the DO mechanism is $E<10^{-5}$.

For network (20) with undelayed couplings, CS is realized effectively [Fig.5]. From Eqs. $(22,23)$, all oscillators have uniform intensities during the transition to synchronization, regardless of heterogeneous degrees. But this is totally different from adaptive networks $[21,27]$. The average intensity $S(k)$ over oscillators with degree $k$ increases as $S(k) \sim k^{\beta}$ with $\beta \sim 0.5$ [27].

After the adaptation, network (20) with undelayed couplings can be rewritten as $\dot{\mathbf{x}}_{i} \approx \mathbf{F}\left(\mathbf{x}_{i}\right)+S_{0}\left[\mathbf{H}_{0}\left(\overline{\mathbf{x}}_{i}\right)-\mathbf{H}_{0}\left(\mathbf{x}_{i}\right)\right]$, where $S_{0}=\varepsilon n_{0}$ is the ultimate intensity, $n_{0}$ is the ending adjustment step, and $\overline{\mathbf{x}}_{i}=\left(1 / k_{i}\right) \sum_{j \in K_{i}} \mathbf{x}_{j}$ is the local mean field of neighbors [32]. In randomly enough networks the local mean field $\overline{\mathbf{x}}_{i}$ of oscillators with $k_{i} \gg 1$ can be approximated by the global mean field $\overline{\mathbf{x}}_{i}=\overline{\mathbf{x}}$. Hence we get $\dot{\mathbf{x}}_{i} \approx$ $\mathbf{F}\left(\mathbf{x}_{i}\right)+S_{0}\left[\mathbf{H}_{0}(\overline{\mathbf{x}})-\mathbf{H}_{0}\left(\mathbf{x}_{i}\right)\right]$. Hence all oscillators are forced by a common mean field signal $\mathbf{H}_{0}(\overline{\mathbf{x}})$ with the same forcing strength $S_{0}$, and all oscillators synchronize at a similar speed to the mean activity $\overline{\mathbf{x}}$. The speed only depends on the same intensity (i.e. the sum of input signals each oscillator receives), regardless of the network size. The independence of the network size is not satisfied in Refs. [21,27], where the speed strongly relies on heterogeneous intensities.

For the network (20) with undelayed couplings, the adaptive strategies can realize CS both in SF networks with undelayed couplings and in SW networks with undelayed couplings [43]. However, even for SW networks with homogeneous degrees, the adaptive strategies can not ensure uniform intensities if all oscillators have different initial conditions. We plot the intensities $S_{i}$ (i.e. $S_{i}=\sum_{j \in K_{i}} G_{i j}$ ), for 20 arbitrarily chosen oscillators in SW networks according to the Zhou-Kurths method [Fig.6(a)]. When the adaptation parameter is chosen as $\gamma=0.002$, we find that the Zhou-Kurths method can not ensure uniform intensities during or after the adaptation. Based on the DO mechanism, synchronization in SW networks is realized effectively [Fig.7(a)], and the intensities are always uniform during the transition to synchronization. From Fig.7(b), the intensity $S=S_{i}$ is also a good indicator for 

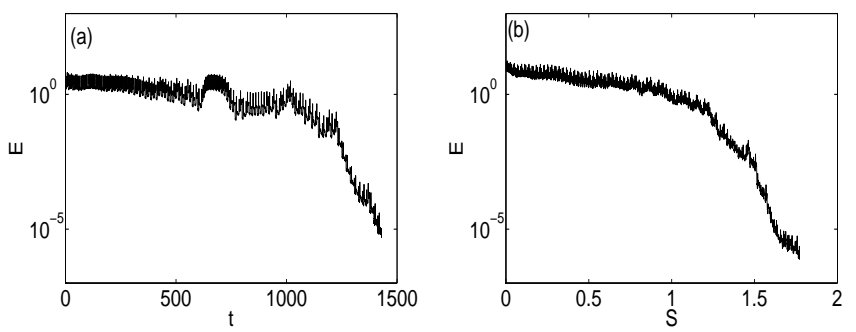

Fig. 7 The average synchronization error $E$ in SW networks with undelayed couplings as a function of (a) time $t$, and (b) intensity $S$, by the DO mechanism. The parameters are $N=500, K=4$, $p=0.003, T=1 \mathrm{~s}, \varepsilon=0.001$.

synchronization in networks. As $S$ increases to a critical value, a network becomes synchronous.

For the network (20) with delayed couplings, even for a small time delay $\tau$ (such as $\tau=0.01 \mathrm{~s}$ ), the Zhou-Kurths method can not realize synchronization in SW networks [Fig.8(a)]. The synchronization error between two connected oscillators is about $10^{-2} \times 500=5$ for networks with $N=500$. Due to the DO mechanism, synchronization can be realized effectively when the time delay $\tau=2 \mathrm{~s}$ [Fig.8(b)]. The synchronization error is about $10^{-5} \times 500=0.005$. Hence the DO mechanism is much more effective than the Zhou-Kurths method. The main reason is that the DO mechanism enures that the intensities are always uniform during the transition to synchronization. But the Zhou-Kurths method can not ensure uniform intensities even for a small time delay [Fig.6(b)]. Though the difference of intensities between oscillators is small initially, it becomes large as time increases. The uniformity of intensities is a necessary condition for the existence of a synchronous manifold in NW networks with delayed couplings. After the adaptation, the synchronous manifold is given by $\left\{\mathbf{x}_{i}(t)=\mathbf{x}_{0}(t), i=1, \cdots, N\right\}$, where $\mathbf{x}_{0}(t)$ is the solution of the isolated dynamics

$$
\dot{\mathbf{x}}_{0}(t)=\mathbf{F}\left(\mathbf{x}_{0}(t)\right)+S_{0}\left(\mathbf{H}_{0}\left(\mathbf{x}_{0}(t-\tau)\right)-\mathbf{H}_{0}\left(\mathbf{x}_{0}(t)\right)\right) .
$$

Remark: The DO mechanism can be also applied to PS in networks with nonidentical oscillators, provided that the phase in networks of oscillators is welldefined [6]. For the Kuramoto model, the accumulated synchronization error (21) is defined by

$$
E_{n}(i, j)=\frac{1}{T} \int_{t_{n-1}}^{t_{n}}\left[1-r_{n}(i, j)\right] d t
$$

with $r_{n}(i, j) \mathrm{e}^{\zeta \Psi_{n}(i, j)}=\left(\mathrm{e}^{\zeta \theta_{j}}+\mathrm{e}^{\zeta \theta_{i}}\right) / 2$, where $0 \leq r_{n}(i, j) \leq 1$ measures the extent of synchronization of oscillators $i, j$, and $\Psi_{n}(i, j)$ stands for an average phase.

For the networks $\dot{\mathbf{x}}_{i}=\tau_{j} \mathbf{F}_{i}\left(\mathbf{x}_{i}\right)+\sum_{j=1}^{N} G_{i j} \mathbf{H}\left(\mathbf{x}_{j}, \mathbf{x}_{i}\right)$, where the parameter $\tau_{j}$ is distributed uniformly in an interval $[1-\triangle \tau, 1+\triangle \tau]$ with the paprameter $\triangle \tau=0.1$, the accumulated synchronization error (21) is defined by 

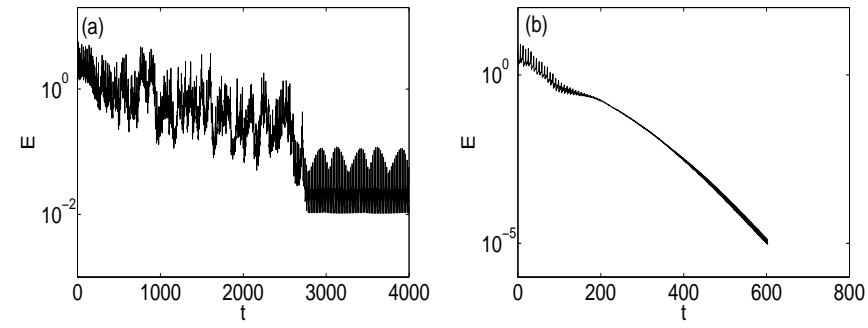

Fig. 8 The average synchronization error $E$ in SW networks with delayed couplings as a function of time $t$. (a) the Zhou-Kurths method ( $\tau=0.01 \mathrm{~s})$. (b) the DO mechanism $(\tau=2 \mathrm{~s})$. The parameters $N=500, p=0.003, \gamma=0.002, T=1 \mathrm{~s}, \varepsilon=0.001$.

$$
E_{n}(i, j)=\frac{1}{T} \int_{t_{n-1}}^{t_{n}}\left[1-r_{n}(i, j)\right] d t
$$

where $r_{n}(i, j) \mathrm{e}^{\zeta \Psi_{n}(i, j)}=\left(\mathrm{e}^{\zeta \vartheta_{j}}+\mathrm{e}^{\zeta \vartheta_{i}}\right) / 2$, the phase $\vartheta_{i}$ can be simply defined by $\vartheta_{i}=$ $\left.\arctan \left(y_{i} / x\right) i\right)$ [6]. Of course, for the above two cases, we should choose suitable ending conditions (such as Eq. (15) and $\left|r_{\text {link }}^{\prime}\left(n_{0}\right)-1\right|<10^{-2}$ ).

Note that the DGN approach can be also applied to PS in networks with much more complex non-identical oscillators, such as the networks of Rössler oscillators. In this case, the order parameters $r(t), r_{i}(t), r_{\text {link }}, r_{\text {link }}^{i, n}$ are defined according to the phase $\vartheta_{i}$.

\subsection{Enhanced synchronizability in ACNs}

We first briefly review the stability of networks with one time-invariant topology:

$$
\dot{\mathbf{x}}_{i}=\mathbf{F}\left(\mathbf{x}_{i}\right)+\sigma \sum_{j=1}^{N} G_{i j}^{0} \mathbf{H}_{0}\left(\mathbf{x}_{j}\right), 1 \leq i \leq N,
$$

where $\sigma$ is the overall strength, $\mathbf{F}\left(\mathbf{x}_{i}\right)$ is the dynamics of individual oscillator, $\mathbf{H}_{0}\left(\mathbf{x}_{j}\right)$ is the output function. For a generally asymmetric matrix $G^{0}=\left(G_{i j}^{0}\right)$ with $G_{i j}^{0}=$ $W_{i j}^{0} A_{i j}$, the variational equation for the synchronous state $\left\{\mathbf{x}_{i}=\mathbf{s}, \forall i\right\}$ is

$$
\dot{\xi}_{i}=\left[D \mathbf{F}_{0}(\mathbf{s})-\sigma \lambda_{l} D \mathbf{H}_{0}(\mathbf{s})\right] \xi_{i}
$$

where $D$ is the Jacobian operator, $\lambda_{l}$ are the complex eigenvalues of the Laplacian matrix $L\left(=-G^{0}\right)$, satisfying $\operatorname{Re}\left(\lambda_{1}\right) \leq \operatorname{Re}\left(\lambda_{2}\right) \leq \cdots \leq \operatorname{Re}\left(\lambda_{N}\right)$. The largest Lyapunov exponent (LLE), $\Lambda(\alpha, \beta)$, of

$$
\dot{\eta}=\left[D \mathbf{F}_{0}(\mathbf{s})-(\alpha+i \beta) D \mathbf{H}_{0}(\mathbf{s})\right] \eta
$$




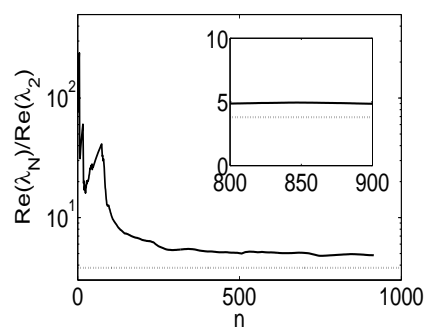

Fig. 9 The ratio $\operatorname{Re}\left(\lambda_{N}\right) / \operatorname{Re}\left(\lambda_{2}\right)$ as a function of the adjustment step $n$ in SF networks. Solid line: CS in network (26) with $G^{0}=G$; dotted line: $R_{\text {opt }}$. Inset: the stationary ratio. The parameters are $N=1000, M=5, T=1 \mathrm{~s}$ and $\varepsilon=0.001$.

is a function of $\alpha$ and $\beta$, which is the master stability function (MSF) [17,18]. Let $\mathscr{R}$ be the region in the complex plane where the MSF provides a negative LLE. The condition for CS in network (26) is that the set $\left\{\sigma \lambda_{l}, \forall l\right\}$ is entirely contained in $\mathscr{R}$ [17]. Here we only consider the case where the region $\mathscr{R}$ is bounded. Then, a better synchronizability is achieved if simultaneously the ratio $\operatorname{Re}\left(\lambda_{N}\right) / \operatorname{Re}\left(\lambda_{2}\right)$ and $\max \left|\operatorname{Im}\left(\lambda_{i}\right)\right|$ are minimized [33,38].

For SF networks, the DO mechanism realizes CS in network (20) effectively. During the transition to synchronization in network (20), the ratio $\operatorname{Re}\left(\lambda_{N}\right) / \operatorname{Re}\left(\lambda_{2}\right)$ in network (26) with $G^{0}=G$ approaches the optimal synchronizability $R_{\mathrm{opt}}=3.8$ [Fig. 9]. The value $R_{\text {opt }}$ is determined by the coupling matrix $G^{\prime}(\alpha)=\left(G_{i j}^{\prime}(\alpha)\right)$ with $G_{i j}^{\prime}(\alpha)=\left(k_{i} k_{j}\right)^{\alpha} / \sum_{j \in K_{i}}\left(k_{i} k_{j}\right)^{\alpha}$ and $G_{i i}^{\prime}(\alpha)=-1$, which extends the couplings in networks [39]. When $\alpha=0$, the eigenratio of the Laplacian matrix of $G^{\prime}(0)$ is minimal and the synchronizability in network (26) with $G^{0}=G^{\prime}(0)$ is optimal [39]. From Eqs. (21-23), the incoming link to be adjusted for each oscillator is always chosen to be the pair of oscillators with the maximal synchronization difference in the previous time interval, which substantially decreases the ratio $\operatorname{Re}\left(\lambda_{N}\right) / \operatorname{Re}\left(\lambda_{2}\right)$. From Fig. 9, this is a dynamical process towards the optimal synchronizability $R_{\text {opt }}$.

Here we assign the coupling matrix $G^{0}$ in network (26) by

$$
G^{0}=G_{\text {norm }}=G_{\text {end }} / S
$$

where $G_{\text {end }}$ is the coupling matrix of network (20) after the adaptation. Since all oscillators have uniform intensities, the Laplacian matrices of $G_{\text {norm }}$ and $G_{\text {end }}$ have equal ratios $\operatorname{Re}\left(\lambda_{N}\right) / \operatorname{Re}\left(\lambda_{2}\right)$. The ratio $\operatorname{Re}\left(\lambda_{N}\right) / \operatorname{Re}\left(\lambda_{2}\right)$ in network (26) with $G^{0}=G_{\text {norm }}$ is shown by the stationary value [Fig.9: Inset]. When $\sigma=1.5$, all nonzero eigenvalues of the Laplacian matrix of $\sigma G_{\text {norm }}$ are located in a very narrow region around the real axes in the region $\mathscr{R}$, and the absolute values of imaginary parts are sufficiently small [Fig.10]. Hence the ratio $\operatorname{Re}\left(\lambda_{N}\right) / \operatorname{Re}\left(\lambda_{2}\right)$ is a good indicator for the synchronizability in network (26). In this section the synchronizability in network (26) with $G^{0}=G_{\text {norm }}$ is quasi-optimal, compared with the optimal synchronizability [39]. 

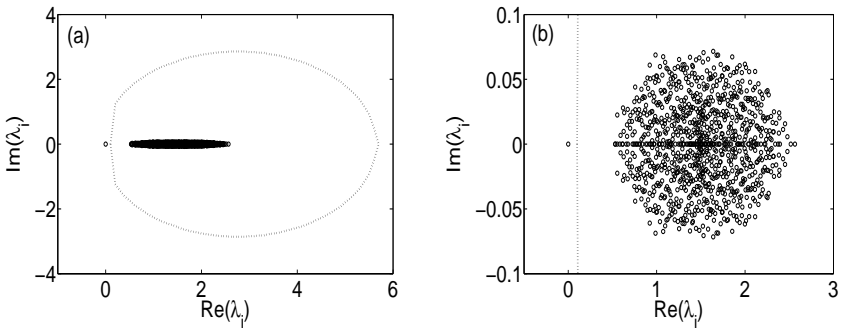

Fig. 10 (a,b) Distribution of eigenvalues of the Laplacian matrix of $\sigma G_{\text {norm }}$ in SF networks. Circle: CS in network (26) with $G^{0}=G_{\text {norm }}$; solid line: the region $\mathscr{R}$. The parameters are $N=1000, M=5$, $T=1 \mathrm{~s}, \varepsilon=0.001$ and $\sigma=1.5$.
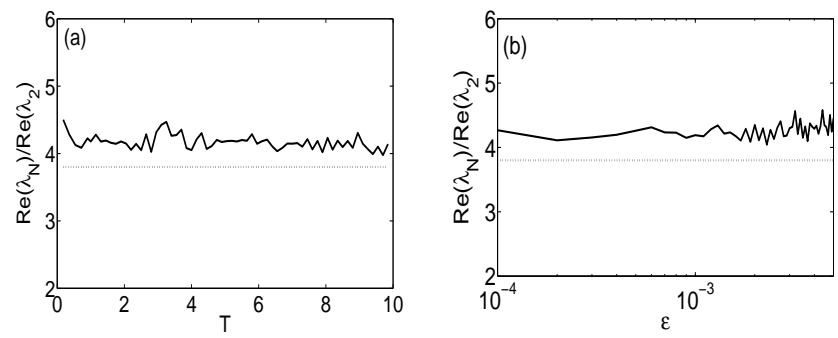

Fig. 11 (a,b) The ratio $\operatorname{Re}\left(\lambda_{N}\right) / \operatorname{Re}\left(\lambda_{2}\right)$ for different $T$ (a) and $\varepsilon$ (b), in SF networks with $N=1000$, $M=5$. Solid line: CS in network (26) with $G^{0}=G_{\text {norm }}$; dotted line: $R_{\mathrm{opt}}$. All the estimates are averaged over 20 realizations of networks.

We discuss the effect of the parameters $T$ and $\varepsilon$ on the synchronizability in net-

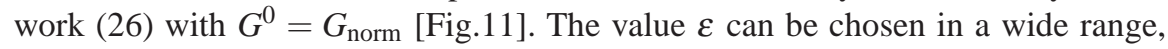
and the length $T$ can be arbitrary large. In our simulations $\varepsilon$ is from [0.0001,0.005]. From Fig.11, the ratio $\operatorname{Re}\left(\lambda_{N}\right) / \operatorname{Re}\left(\lambda_{2}\right)$ is almost independent of the values of $T$ and $\varepsilon$.

The ratio $\operatorname{Re}\left(\lambda_{N}\right) / \operatorname{Re}\left(\lambda_{2}\right)$ in network (26) with $G^{0}=G_{\text {norm }}$ increases slightly with increasing network size $N$, and can be well-fitted by a power-law dependence, i.e. the synchronizability decreases slightly [Fig.12]. From the fitting, we find that the network (26) is still synchronizable till $N \approx 10^{11}$. In this section, the size of the network (26) that is synchronizable exceeds by several orders of magnitude the size of unweighted networks $\left(\approx 10^{3}\right)$ and networks with adaptive couplings $\left(\approx 8 \times 10^{5}\right)$ [27]. Obviously, this is a great enhancement of the synchronizability in networks, compared with unweighted networks and networks with adaptive couplings [27]. It should be pointed out that for different size of networks, $\max \left|\operatorname{Im}\left(\lambda_{i}\right)\right|$ is sufficiently small (the maximal value is less than 0.06).

The above result can be ensured by the Gerschgorin disk theorem [41]. For the coupling matrix $G^{0}=G_{\text {norm }}$, all eigenvalues are fully contained within the unit circle centered at 1 . So $0 \leq \operatorname{Re}\left(\lambda_{l}\right) \leq 2,\left|\operatorname{Im}\left(\lambda_{l}\right)\right| \leq 1$, and the largest $\operatorname{Re}\left(\lambda_{N}\right)$ will never diverge, independently of the network size $N$ [38]. During the transition to synchro- 


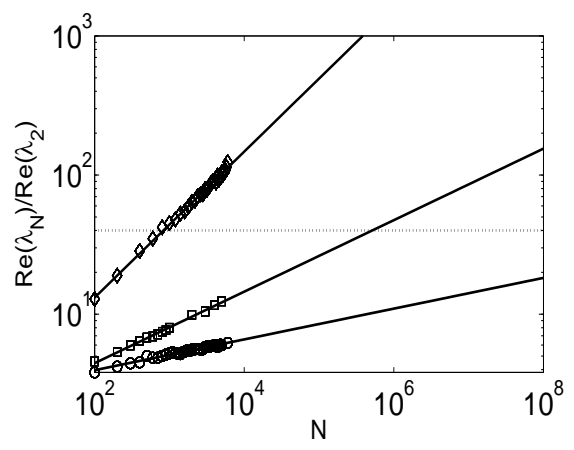

Fig. 12 The ratio $\operatorname{Re}\left(\lambda_{N}\right) / \operatorname{Re}\left(\lambda_{2}\right)$ for different size of SF network (26) with $M=5, \tau=1, \varepsilon=$ 0.001. Diamond: unweighted networks; square: networks with adaptive couplings [17]; circle: CS; fitting: solid line; dotted line: $R_{\varepsilon}=40$ (the maximal ratio $\operatorname{Re}\left(\lambda_{N}\right) / \operatorname{Re}\left(\lambda_{2}\right)$ in the region $\mathscr{R}$ ). All the estimates are averaged over 20 realizations of networks.

nization in network (20), $S_{\max } / S_{\min }$ always equals to 1 . In Refs. [27,32], the synchronizability decreases with the increasing of $S_{\max } / S_{\min }$, but $S_{\max } / S_{\min }$ increases with the increasing of the size $N$. Hence the synchronizability here is better than the synchronizability in Ref. [27], whose main aim is to reduce the heterogeneity of intensities adaptively.

From the above analysis, we find that the DO mechanism results in a better synchronizability in SF networks, compared with unweighted networks and adaptive networks. Now we also discuss the synchronizability in SW networks due to the DO mechanism.

Obviously, the synchronization in SW networks can be realized by the DO mechanism. Similarly, we assign the coupling matrix $G^{0}$ in SW networks by Eq.(29), after the adaptation. In order to enhance synchronizability in SW networks, we compare the synchronizability in the unweighted network (26) (type I network: $W_{i j}^{0}=1$ ), the degree based weighted network (26) (type II network: $W_{i j}^{0}=1 / k_{i}$ ), network (26) with adaptive couplings by the Zhou-Kurths method (type III network), and network (26) with the coupling matrix being designed by network (20) with undelayed couplings (type IV networks).

We find that for a fixed small probability $p$ (such as $p=0.003$ ) for adding longrange connections, the synchronizability in type III networks is better than that in type I networks, but it is worse than that in type II networks, no matter how large the size $N$ of the networks is [Fig.13(a)]. However, we find that type IV networks have a better synchronizability than both type II and type III networks when the size is not too large. Of course, the smaller the probability $p$ is, the larger is the size of type IV networks with better synchronizability than both type II and type III networks. For the fixed size $N=500$, we observe similar results in a certain range of the probability $p$ [Fig.13(b)]. From Fig.13, we see that the synchronizability in type IV networks is better than those in type II networks and type III networks in some cases. It is reasonable that type IV networks have better synchronizability than 

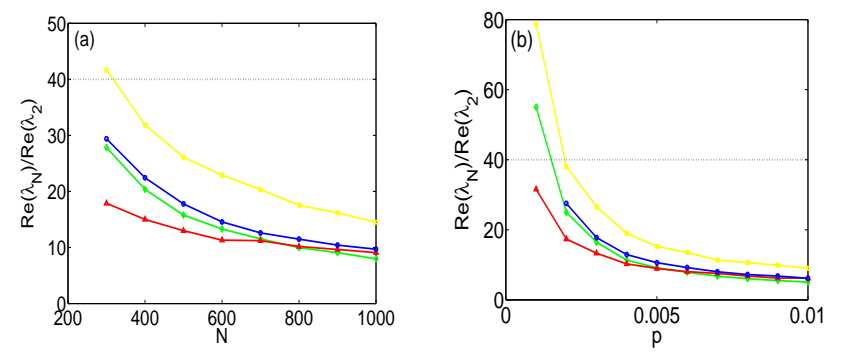

Fig. 13 For SW networks, the ratio $\operatorname{Re}\left(\lambda_{N}\right) / \operatorname{Re}\left(\lambda_{2}\right)$ as a function of the network size $N$ for a fixed probability $p=0.003$ (a), and the probability $p$ for a fixed size $N=500$ (b). Yellow line (square): type I networks; green line (diamond): type II networks; blue line (circle): type III networks; red line (big triangle up): type IV networks; black dashed line: the maximal ratio $\frac{\operatorname{Re}\left(\lambda_{N}\right)}{\operatorname{Re}\left(\lambda_{2}\right)}$ in the region $\mathscr{R}$. The parameters are $K=4, \gamma=0.002, T=1 \mathrm{~s}, \varepsilon=0.001$. All the estimates are averaged over 20 realizations of networks.

type III networks. This is because the DO mechanism ensures uniform intensities of all oscillators in type IV networks. Now we further analyze the reason why type IV networks have better synchronizability than type II networks in a certain range of the probability $p$.

In order to do so, we slightly modify SW networks. The initial network is a $K$-nearest-neighbor coupled network consisting of $N$ oscillators arranged in a ring, with each oscillator $i$ being adjacent to its $K$ neighbor oscillators $i \pm 1, \cdots, i \pm K / 2$, and with $K$ being even. Then one adds with probability $p$ a long-range connection between a pair of oscillators with indices satisfying

$$
n_{1} \leq \min \{|i-j|, N-|i-j|\} \leq n_{2},
$$

where $0 \leq n_{1}, n_{2} \leq N / 2$ are two positive integers. This kind of networks is called type $\mathrm{V}$ networks. Based on type $\mathrm{V}$ networks, we adjust the coupling strengths by the DO mechanism. After the adaptation, we define the average coupling strength $\left\langle W_{v}\right\rangle$ over the $k_{W}$ links having the same $v=\min \{|i-j|, N-|i-j|\}$ :

$$
\left\langle W_{v}\right\rangle=\frac{1}{k_{W}} \sum G_{i j} .
$$

Further, for unweighted type $\mathrm{V}$ networks, the average load $\left\langle L_{v}\right\rangle$ over the $k_{L}$ links having the same $v$ is given by

$$
\left\langle L_{v}\right\rangle=\frac{1}{k_{L}} \sum L_{i j}
$$

where the load $L_{i j}$ of the link connecting oscillators $i$ and $j$ quantifies the traffic of the shortest paths passing that link. Here the size of type $\mathrm{V}$ networks is $N=300$ and the probability $p=0.2$. For different $n_{1}$ and $n_{2}$, we plot the relationship between $\left\langle W_{v}\right\rangle$ and $v$ [Fig.14(a,d,g)], and the relationship between $\left\langle L_{v}\right\rangle$ and $v$ [Fig.14(b,e,h)], 

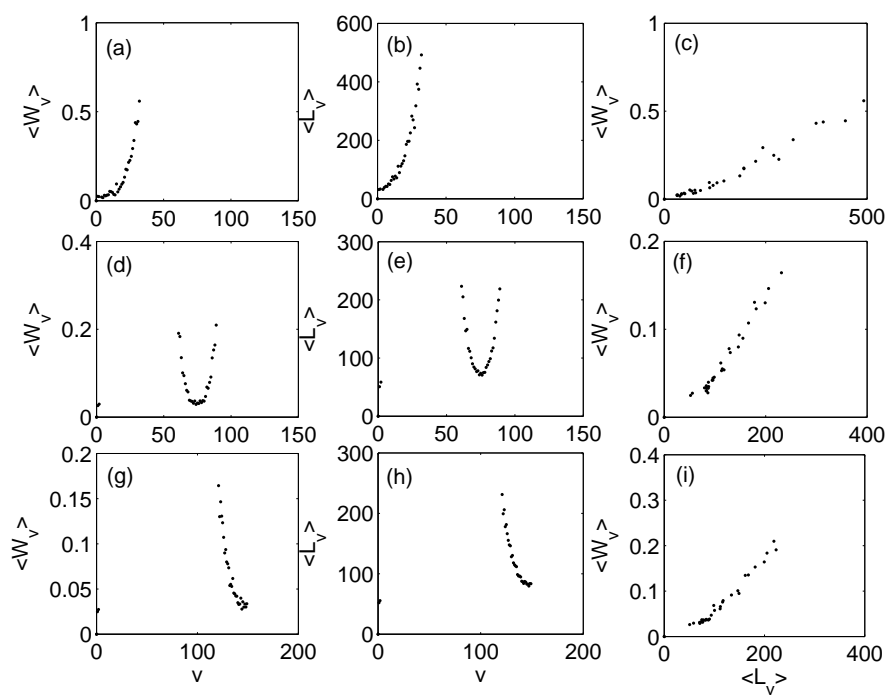

Fig. 14 The dependence of $\left\langle W_{v}\right\rangle$ on $v$ in (a,d,g), of $\left\langle L_{v}\right\rangle$ on $v$ in (b,e,h), and the relationship between $\left\langle W_{v}\right\rangle$ and $\left\langle L_{v}\right\rangle$ in $(c, f, i)$, respectively. $n_{1}=0, n_{2}=30(\mathrm{a}, \mathrm{b}, \mathrm{c}) ; n_{1}=60, n_{2}=90(\mathrm{~d}, \mathrm{e}, \mathrm{f}) ; n_{1}=120$, $n_{2}=150(\mathrm{~g}, \mathrm{~h}, \mathrm{i})$. The parameters in type V networks are $N=300, K=4, p=0.2, T=1 \mathrm{~s}, \varepsilon=$ 0.001 .

respectively. From these subfigures, we conclude that $\left\langle W_{v}\right\rangle$ has a similar dependence on $v$ as $\left\langle L_{v}\right\rangle$, which is further verified by the relationship $\left\langle W_{v}\right\rangle \sim\left\langle L_{v}\right\rangle$ [Fig.14(c,f,i)]. This implies that the adaptation due to the DO mechanism may lead to a similar synchronizability as the load based weighted networks. This may in part explain why type IV networks have a better synchronizability than type II networks in a certain range of the probability $p$ for adding long-range connections.

Remarks: From the above subsection, we can extend the DO mechanism to CS in network (20) with the coupling function $\mathbf{H}\left(\mathbf{x}_{j}, \mathbf{x}_{i}\right)=\mathbf{H}\left(\mathbf{x}_{j}\left(t-\tau_{0}\right)\right)-\mathbf{H}\left(\mathbf{x}_{i}\right)$ and a small delay time $\tau_{0}$ (such as $\tau_{0} \leq 2$ ). The DO mechanism ensures that all oscillators have uniform intensities, which leads to the existence of a synchronous manifold in network (20). However, it can not be realized by the dynamical mechanism proposed in Ref. [27]. Due to the DO mechanism, we can also obtain a better synchronizability in SF and SW networks due to CS in networks with delayed couplings. Here we omit the corresponding results.

From the DGN approach and the DO mechanism, the two coupling schemes are "winner-take-all" strategies. This implies that the intensity $S_{i}$ for oscillator $i$ increases to infinity as the adjustment time $n$ tends to infinity. Hence there is one shortcoming: we should choose suitable conditions to end the adaptation of the above two mechanisms. In fact, this shortcoming can be overcome by slightly modifying the adjustment $(8,9)$ for the DGN approach and the adjustment $(23)$ for the DO mechanism. Here the adjustment of couplings for the incoming link with the maximal 
competition ability is modified as follows:

$$
W_{i \mu_{i}^{n}}^{n+1}:=W_{i \mu_{i}^{n}}^{n}+\varepsilon \mathrm{e}^{-n / k_{0}}
$$

for the DGN approach, and

$$
W_{i j_{\max }^{n}}^{n+1}=W_{i j_{\max }^{n}}^{n}+\varepsilon \mathrm{e}^{-n / k_{0}}
$$

for the DO mechanism, where $k_{0}$ is a suitable positive integer. From Eqs. $(33,34)$, the intensity $S_{i}$ still increases, and all oscillators have uniform intensities. However, the intensity $S_{i}$ for each oscillator can not increase to infinity, and can be bounded by the limit $\bar{S}=\lim _{n \rightarrow \infty} S_{i}$ for all oscillators, where

$$
\bar{S}=\varepsilon \mathrm{e}^{-1 / k_{0}} /\left(1-\mathrm{e}^{-1 / k_{0}}\right) .
$$

Obviously, we can adjust the ultimate intensity for all oscillators by a suitable parameter $k_{0}$. When $k_{0}$ is larger, the intensity $\bar{S}$ is larger; when $k_{0}$ is smaller, the intensity $\bar{S}$ is also smaller. It should be noted that we obtain similar results if we choose the parameters $k_{0}=500, \varepsilon=0.01$ for PS in the Kuramoto models and $k_{0}=1000, \varepsilon=0.001$ for CS in networks of Rössler oscillators, respectively.

\section{Conclusions}

In this chapter, we introduce two dynamical optimization coupling mechanisms for getting different kinds of synchronization in adaptive complex networks, whose oscillators could be either identical or non-identical. For each oscillator, we adjust only one incoming link's strength in different time intervals while the other incoming links' strengths remain constant. The dynamical optimization coupling mechanisms are in effect "winner-take-all" strategies. If one incoming link for each oscillator has the maximal competition ability in its neighborhood in different time intervals, its strength increases by a small value. We realize different kinds of synchronization in adaptive complex networks with undelayed or delayed couplings, as well as ensure that all oscillators have uniform intensities during the transition to synchronization. We also enhance the synchronizability in complex networks with identical oscillators.

Acknowledgements M.C. was supported by the Alexander von Humboldt Foundation and Special Doctoral Fund at Tsinghua University by Ministry of Education under Grant. No. 20070003129. J.K. was supported by SFB 555 (DFG) and BRACCIA (EU). 


\section{References}

1. Watts, D.J., Strogatz, S.H.: Collective dynamics of 'small world' networks. Nature (London) 393, 440-442 (1998).

2. Barabasi, A.L., Albert, R.: Emergence of scaling in random networks. Science 286, 509-512 (1999).

3. Strogatz, S.H.: Exploring complex networks. Nature (London) 410, 268-276 (2001).

4. Albert, R., Barabasi, A.L.: Statistical mechanics of complex networks. Rev. Mod. Phys. 74, 47-97 (2002).

5. Boccaletti, S., Latorab, V., Morenod, Y., Chavez, M., Hwang, D.U.: Complex networks: structure and dynamics. Phys. Rep. 424, 175-308 (2006).

6. Osipov, G., Kurths, J., Zhou, C.S.: Synchronization in oscillatory networks. Spring-Verlag (2007).

7. Kuramoto, Y.: Chemical Oscillations, Waves, and Turbulence. Springer, Berlin (1984).

8. Acebron, J., Bonilla, L.L., Vicente, C.J.P., Ritort, F., Spigler, R.: The Kuramoto model: a simple paradigm for synchronization phenomena. Rev. Mod. Phys. 77, 137-185 (2005).

9. Strogatz, S.H.: From Kuramoto to Crawford: exploring the onset of synchronization in populations of coupled oscillators. Physica D 143, 1-20 (2000).

10. Jain, S., Krishna, S.: A model for the emergence of cooperation, interdependence, and structure in evolving networks. Proc. Natl. Acad. Sci. U.S.A. 98, 543-547 (2001).

11. Skyrms, B., Pemantle, R.: A dynamic model of social network formation. Proc. Natl. Acad. Sci. U.S.A. 97, 9340-9346 (2000).

12. Zimmermann, M.G., Eguíluz, V.M., Miguel, M.S.: Coevolution of dynamical states and interactions in dynamic networks. Phys. Rev. E 69, 065102 (2004).

13. Ito, J., Kaneko, K.: Spontaneous structure formation in a network of chaotic units with variable connection strengths. Phys. Rev. Lett. 88, 028701 (2002).

14. Gleiser, P.M., Zanette, D.H.: Synchronization and structure in an adaptive oscillator network. European Phys. J. B 53, 233-238 (2006).

15. Gross, T., Blasius, B.: Adaptive aoevolutionary networks-a review. arXiv:0709.1858v1 [physics.soc-ph] 12 Sep 2007.

16. Newman, M.E.J., Watts, D.J.: Renormalization group analysis of the small-world network model. Phys. Lett. A 263, 341-346 (1999).

17. Pecora, L.M., Carroll, T.L.: Master stability functions for synchronized coupled systems. Phys. Rev. Lett. 80, 2109-2112 (1998).

18. Barahona, M., Pecora, L.M.: Synchronization in small-world systems. Phys. Rev. Lett. 89, 054101 (2002).

19. Nishikawa, T., Motter, A.E., Lai, Y.C., Hoppensteadt, F.C.: Heterogeneity in oscillator networks: are smaller worlds easier to synchronize? Phys. Rev. Lett. 91, 014101 (2003).

20. Maistrenko, Y.L., Lysyansky, B., Hauptmann, C., Burylko, O., Tass, P.A.: Multistability in the Kuramoto model with synaptic plasticity. Phys. Rev. E 75, 066207 (2007).

21. Ren, Q., Zhao, J.: Adaptive coupling and enhanced synchronization in coupled phase oscillatorsPhys. Rev. E 76, 016207 (2007).

22. Chen, M., Shang, Y., Zou, Y., Kurths, J.: Synchronization in the Kuramoto model: a dynamical gradient network approach. Phys. Rev. E. 77, 027101 (2008).

23. Wang, X., Lai, Y.C., Lai, C.H.: Enhancing synchronization based on complex gradient networks. Phys. Rev. E 75, 056205 (2007).

24. Moreno, Y., Pacheco, A.F.: Synchronization of Kuramoto oscillators in scale-free networks. Europhys. Lett. 68, 603-609 (2004).

25. Arenas, A., Díaz-Guilera, A., Pérez-Vicente, J.C.: Synchronization reveals topological scales in complex networks. Phys. Rev. Lett. 96, 114102 (2006).

26. Gómez-Garden̈es, J., Moreno, Y., Arenas, A.: Paths to synchronization on complex networks. Phys. Rev. Lett. 98, 034101 (2007).

27. Zhou, C., Kurths, J.: Dynamical weights and enhanced synchronization in adaptive complex networksPhys. Rev. Lett. 96, 164102 (2006). 
28. Lu, W.L.: Adaptive dynamical networks via neighborhood information: synchronization and pinning control. Chaos 17, 023122 (2007).

29. Huang, D.B.: Synchronization in adaptive weighted networks. Phys. Rev. E 74, 046208, (2006).

30. Sorrentino, F., Ott, E.: Adaptive synchronization of dynamics on evolving complex networks. arXiv:0802.1241v1 [cond-mat.dis-nn] 10 Feb 2008.

31. Frasca, M., Buscarino, A., Rizzo, A., Fortuna, L., Boccaletti, S.: Synchronization of moving chaotic agents. Phys. Rev. Lett. 100, 044102 (2008)

32. Zhou, C., Motter, A.E., Kurths, J.: Universality in the synchronization of weighted random networks. Phys. Rev. Lett. 96, 034101 (2006).

33. Huang, D., Chavez, M., Amann, A., Boccaletti1, S.: Synchronization in complex networks with age ordering. Phys. Rev. Lett. 94, 138701 (2005).

34. Radicchi, F., Meyer-Ortmanns, H.: Reentrant synchronization and pattern formation in pacemaker-entrained Kuramoto oscillators. Phys. Rev. E 74, 026203 (2006).

35. Park, K., Lai, Y.C., Zhao, L., Ye, N.: Jamming in complex gradient networks. Phys. Rev. E 71, 065105(R) (2005).

36. Toroczkai, Z., Bassler, K.E.: Jamming is limited in scale-free systems. Nature 428, 716-716 (2004).

37. Chavez, M., Hwang, D.U., Amann, A., Hentschel, H.G.E., Boccaletti, S.: Synchronization is enhanced in weighted complex networks. Phys. Rev. Lett. 94, 218701 (2005).

38. Chavez, M., Hwang, D.U., Amann, A., Boccaletti, S.: Synchronizing weighted complex networks. Chaos 16, 015106 (2006).

39. Motter, A.E., Zhou, C.S., Kurths, J.: Network synchronization, diffusion, and the paradox of heterogeneity. Phys. Rev. E 71, 016116 (2005).

40. Nishikawa, T., Motter, A.E.: Synchronization is optimal in nondiagonalizable networks. Phys. Rev. E 73, 065106 (2006).

41. Varga, R.S.: Gersgorin and his circles. Springer-Verlag, Heidelberg (2004).

42. Chen, M., Zhou, C.S., Wu, Y., Kurths, J.: Dynamical optimization and quasi-optimal synchronizability in complex networks. Submitted.

43. Wu, Y., Chen, M., Zhou, C.S., Kurths, J.: Synchronization in small-world networks. Submitted.

44. Kozma, B., Barrat, A.: Consensus formation on adaptive networks. arXiv:0707.4416v1 [physics.soc-ph] $30 \mathrm{Jul} 2007$.

45. Gross, T., Dommar D'Lima, C., Blasius, B.: Epidemica dynamics on an daptive network. Phys. Rev. Lett. 96, 208701 (2006).

46. Shaw, L.B., Schwartz, I.B.: Fluctuating epidemics on adatpive networks. arXiv:0801.0606v1 [q-bio.PE] 3 Jan 2008. 